\title{
Evaluation The Effect of Magnetized green tea extracts on The Bone Density And Osteoporosis In The Experimental Rats By DXA-Scan
}

\author{
Nour Abd alrazaq Hassan $(\mathrm{BSc})^{1}$, Tahseen Hussain Mubarak $(\mathrm{PhD})^{2}$ and Nameer \\ Fadhel Gheaeb $(\mathrm{PhD})^{3}$ \\ Abstract
}

Background: The study showed that magnetized green tea has effects on bone regeneration, bone density and the amount of this effect depends on the intensity of the power and the frequency of the electromagnetic field emitted. The properties of green tea change when exposed to magnetic fields so that its contents change from metals and organic materials and be flexible and easy to penetrate the cell wall and increase the speed of proliferation. This change in properties is necessary for organ growth and the amount of effect depends on the strength of the electromagnetic field and the time of exposure. The study showed that is an improved in quality of the green tea when exposed to the electromagnetic field with significant changes in the minerals and organic matter .Therefore it's penetration into cell wall would be facilitated, which can accelerate ordinary diffusion of green tea. That is vital for growth and development of different organs .Exposing the green tea to strong magnetic fields affect in the green tea mineral content, and its effects depend on the "strength of the magnetic field and exposure time".

Objective: To study on the effect of consumption of magnetized green tea on bone density and osteoporosis in rats.

Patients and Methods: In this study (14) rats were included and these divided into two groups, A and B "7 rats for each group" were included, Group A were fed normal water for 21 days, and group $B$ were fed magnetized green tea for 21 days. The magnetize system that used to magnetized green tea, this consist of 7000 rolls of iron wire around iron tube where was magnetized green tea with 1400 rolls, which is connected to the continuous power supply " $220 \mathrm{~V}$ ". Digital Gauss meter was used to measure the magnetic flux which is equal to $0.9(\mathrm{mT})$. After 21 daily consumption of the magnetized green tea, the bone density was measured by DXA scan device that is used to measure the bone mineral density" BMD", bone mineral content "BMC", T-score and Z-score. Comparing the result of "T-score, Zscore, BMD and BMC" between group A and B of the animals after 21 days of consumption the magnetized green tea.

Results: The study shows an enhancement in the biological activity in rats that consumed the magnetized green tea, by repairing and turnover the bones as compared with other group that used normal water.

Conclusion: It was concluded that the consumptions of magnetized green tea increase the ability of bone repair and turnover.

Keywords: Electromagnetic field ,Densitometry of the bone rats, Magnetized green tea, Osteoporosis ,Bone regeneration.

Corresponding Author: nourabdalrazaq0@ gmail.com 
Received: $7^{\text {th }}$ January 2019

Accepted: $20^{\text {th }}$ February 2019

DOI:https://doi.org/10.26505/DJM.17024430107

\title{
Introduction
}

\author{
${ }^{1,2}$ College of Science - Diyala University - Diyala- Iraq. \\ ${ }^{3}$ College of Medicine - Diyala University - Diyala - Iraq
}

During last Decades there was an increment in the interest in the bio effects of the electromagnetic fields interaction with living organisms. All living organisms are always expose to the electromagnetic fields, Emitted from industrial and. domestic sources, induced by "ELF-MF" on cells function, and the interaction mechanisms of biological systems and electromagnetic fields are still partially understood [8].

Electromagnetic fields consist of waves that transport energy through space characterized by wavelength and frequency, the two of which are inversely correlated "The shorter the wavelength, the greater the frequency". Duration of exposure and strength of the field. ,gradient, rate of change, and orientation along or perpendicular to flow are variously cited as important to the results[5]. The shapes of solute molecules will be modified by physically powerful magnetic fields, leading to precipitated as spherical or round crystals, rather than deposit as sheets, or platelets of hard crystals [9]. Tea as a vital material consists of numerous components and functional groups within the chemical structure, however via ongoing study the basic components that represented some of Tea chemical structure which is Catechin, Caffaien, theanine, and Gallic Acid found, as shown below components structural formulas:

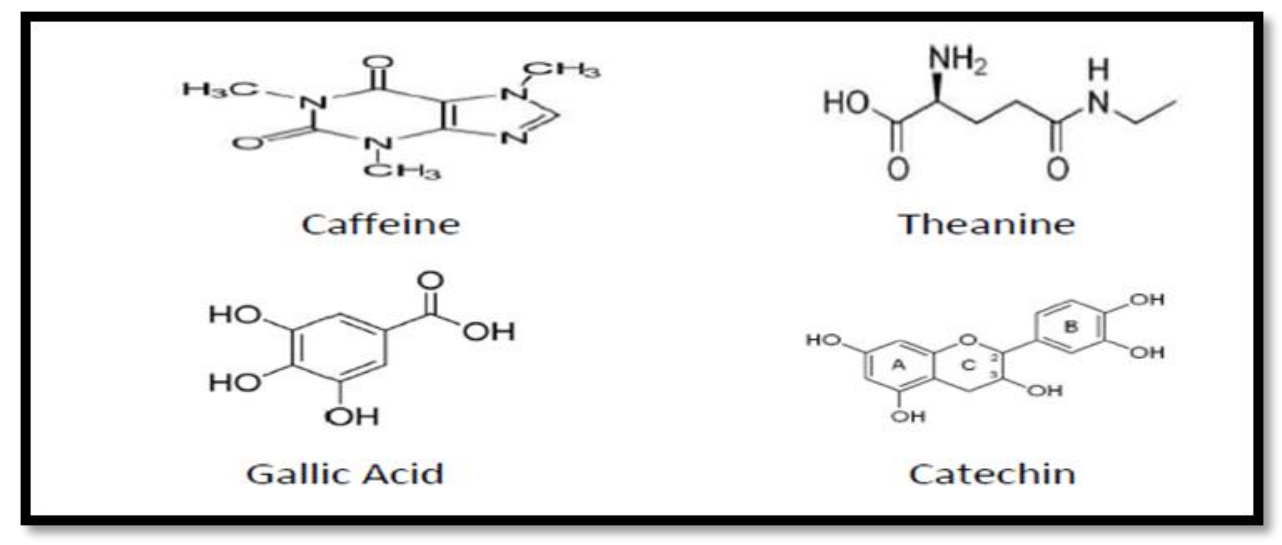

Figure (1): Shows Tea components structural formulas.

"Osteoporosis" is an important systemic in the research's of osteoporosis. disorder, affecting mainly in Caucasian women, with a diverse and multifactorial etiology. wide variety of animal species ,including "rodents, rabbits, dogs, and primates', have been used as animal models "Osteogenesis" is a complex events ,by which" BMSCs (Bone marrow stromal cell)" differentiate to produce new bone. "hBMSCs (Human bone marrow stromal cell)" possess characteristic $\mathrm{Ca} 2+$ waves ,that are involved 
in intracellular signaling. The waves work in short and long periods, the longer periods operating during "trans-cellular signaling"[18].

Bone density measured through "DXA" is an extremely accurate, and accurate method for quantifying bone mineral density "BMD", and mass body composition assessment. "DXA's" primary viable application use to measure the risk to assess fracture ,and diagnosing the osteoporosis.

The osteoporosis diagnosis in (the lumbar spine, proximal hip and, sometimes, the distal forearm are scanned). The whole body also can be scanned to measure whole bone mass and soft tissue of the body composition [13-12].

\section{Patients and Methods}

All procedure were standard by animal ethical committee.An observational non randomized cross-sectional study done from the 1st of December, 2017 to 30th of June 2018, in order to achieve the required objective of the present study and comparing the result with control group to estimate magnetic fields on the intensity of bone turnover process in rats, by means of analysis bone density. The investigations of current study were carried out in Diyala University-
Collage of Medicine . The experiment was performed on rats, males and females adult Wistar rats (Rathus Norvegius) and aged about (6-8) weeks were used in this study. Weight of the animals was between (200$250 \mathrm{gm}$ ) recorded by an electronic balance. The animal procurement from Ministry of Health, Quality Measurement section, laboratory animal house.

Groups study performed on 14 rats the animal were non randomly divided in to two groups A (7 rats for experimental group) and $\mathrm{B}$ (7 rats for control group).

First group (A) was use the magnetized green tea as a treatable material according to the number of rolls using in magnetization 1400, and second group was a control group. Magnetized System

The system of magnetization, an important part of current study in which create magnetic field makes the materials magnetized, is collected, designed, and manufactured manually by the researcher in order to meet the requirements in demand, materials, and specific ratios of the research which approved by supervisors of current study as shown in Figure (2), By using the following As in the Table (1):

\begin{tabular}{|l|l|l||}
\hline \multicolumn{1}{|c|}{ Components } & \multicolumn{1}{c|}{ Specification } \\
\hline \hline 1 & Iron tube open from two ends & length $45 \mathrm{~cm}$ and inner diameter $1.5 \mathrm{~cm}$ \\
\hline 2 & Iron wire & diameter $0.3 \mathrm{~mm}$ \\
\hline \hline 3 & Electric source & $220 \mathrm{~V}$ \\
\hline \hline 4 & Power supply & regulated the voltage supply \\
\hline \hline 5 & Gage (Avometer) & measure the current and voltage \\
\hline \hline 6 & Magnetometer & measurement the flux meter (change field) \\
\hline \hline 7 & Wood box & $(50 * 15) \mathrm{cm}$ \\
\hline \hline 8 & Water faucet & Plastic \\
\hline
\end{tabular}



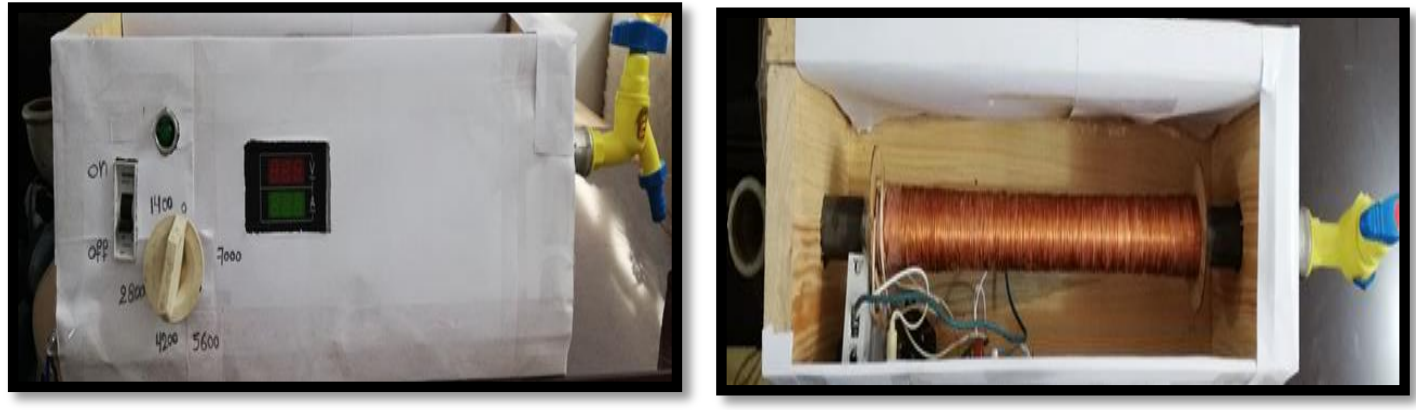

Figure (2): System of magnetization device for required magnetic field

The tube is fixed horizontally inside the wire about 7000 rolls dividing these rolls into wooden box and wrapped up around it iron 5 taps according to data reviled in Table (2):

Table (1): NO. of rolls for each Tap around iron tube and the flux of each tap

\begin{tabular}{|c|c|c||}
\hline No. of Taps & No. of Rolls & The Flux \\
\hline \hline Tap1 & 1400 rolls & $1.5 \mathrm{mT}$ \\
\hline \hline Tap 2 & 2800 rolls & $1.9 \mathrm{mT}$ \\
\hline Tap 3 & 4200 rolls & $0.9 \mathrm{mT}$ \\
\hline Tap4 & 5600 rolls & $0.5 \mathrm{mT}$ \\
\hline Tap 5 & 7000 rolls & $0.3 \mathrm{mT}$ \\
\hline
\end{tabular}

Number of rolls controlled by the channel installed on the wooden box, Where was used 1400 rolls, and the device is connected to the power supply To install the voltage difference using 220 volts in my experience

Table (3): Show the Technical Specifications

\begin{tabular}{|l||l||l||}
\hline & \multicolumn{1}{|c|}{ Feature } & \multicolumn{1}{c|}{ Details } \\
\hline \hline 1 & measuring range & $0 \sim 200 \mathrm{mT} \sim 2000 \mathrm{mT}$ \\
\hline \hline 2 & Accuracy & $\begin{array}{l}0 \sim 100 \mathrm{mT} 1 \%, 100 \mathrm{mT} \text { more than } 2 \% \text { (uniform magnetic field } \\
\text { measurement) }\end{array}$ \\
\hline \hline 3 & Resolution & $-\mathrm{DC} \times 1: 0.00 \sim 200.00 \mathrm{mT} 0.01 \mathrm{mT}$ \\
& & $-\mathrm{DC} \times 10: 0.0 \sim 2000.0 \mathrm{mT} 0.1 \mathrm{mT}$ \\
& & $-\mathrm{AC} \times 1: 0.00 \sim 200.00 \mathrm{mT} 0.01 \mathrm{mT}$ \\
& & $-\mathrm{AC} \times 10: 0.0 \sim 2000.0 \mathrm{mT} 0.1 \mathrm{mT}$ \\
\hline
\end{tabular}

\section{General principle of instrument}

"Accurate flux density" measurement on magnetic and electromagnetic components such as : relays, DC motors, magnets, loudspeakers, etc. It is very relevant for online production testing. As shown in Figure (3). and the whole system is connected to the point of electricity chamber as the system current is direct current DC.

\section{Digital Gauss Meter (china)}


strength of a "magnetic field" is measured in units of (tesla) in the" SI" units, and in (gauss) in the "cgs "system of units. 10,000 gauss are equal to: one tesla [14].

Measuring the magnetization, as a function of temperature and magnetic field .On the base of space, measured the distribution of magnetic field parameters (e.g. amplitude or direction), the magneto vision images that may be generated. Such presentation of magnetic figures is very useful for further investigation and data fusion.

In current study, Digital Gauss Meter connected at electricity chamber has in order to calibrated magnetic flux measurement directly in magnetic field unit Figure(3).

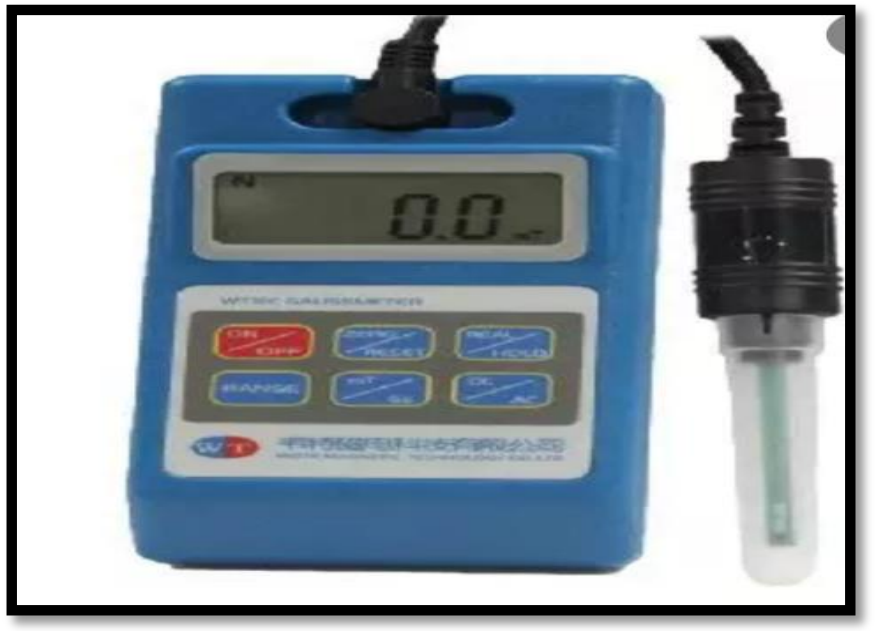

Figure (3) :Digital Gauss Meter for Magnetic Flux Measurement

\section{Procedure /Intervention}

Forty seven babies with NTD were enrolled The whole number of the animals were housed in individual plasticcages with dimension $(40 \times 25 \times 25) \mathrm{cm}$, bedded with wood chips. The animals had at $25^{\circ} \mathrm{C}$, under a 12-12 light /dark cycle and free access to commercial rat pellets (Altromin 1324, Altromin $\mathrm{GmbH}$, Lage, Iraq) and tap water. This was prepare to contain the nutrients that are needed for rapid growth in growing male rats and reproduction in adult female rats based on AIN-93G and AIN-93M, respectively [15].
Rats, were monitor daily for general health, changes in appearance or change in its behavior throughout the study. The rats were concern and maintained in observance, with the direct Care when Use of Laboratory Animals [16] and Massey University guidelines. Ethical approval for the study protocols of rats were gained from Preoperatively, according to Institutional Animal Care, each rat (about 200-250 gm weighting ) has anaesthetized with intentional anesthesia (Ketamine Hydride USP: ketamine 50 mg/ml for i.m./i.v. injection ;Batch NO. 70407 ; Germany), and a dose of general 
analgesia(0.01 $\mathrm{mg} / \mathrm{kg})$ was given intra muscularly. Once the rats reached the required depth of anesthesia, left-sided femur of each selected rat (selection due to highly response of magnetized green tea separately) exposed to DXA scan by researcher under supervision to ensure rats stability during procedure for testing BMD and BMC values affected under specific conditions with magnetized green tea.

\section{Preparation of green tea extracts}

The water was used given to rats in the control group. The green tea was used in the study to obtain normally minerals pre- exist in green tea throughout the experimental period. Green tea extracts were freshly prepared. The green tea were supplied in clean polyethylene green tea bottles. Polyethylene green tea bottles were cleaned weekly. The animals were assigned from groups, the species of study by using the rats about 14 species and dividing it along 2 groups. as show in Table (4):

Table (4): The treatment groups were exposed to magnetized Green tea in 1400 rolls for 21 days

\begin{tabular}{|c|c|}
\hline Group (A) Control & Group (B) Tap (1400) \\
\hline \hline Use normal water ( 7 rats) & Magnetized green tea by use 1400 rolls(7 rats) \\
\hline
\end{tabular}

\section{Magnetic Exposure}

The Green Tea water extracts were freshly prepared by infusing of Green Tea leaves in water for $15 \mathrm{~min}$. The Green Tea solutions were filtered with sterile filter to produce a clear solution and allowed to cool to room temperature prior to serving. Green Tea water extracts was supplied in clean polyethylene water bottles, as the only source of drinking fluid during the study duration. Green Tea water extracts was replaced and served fresh once every day during the feed intake measurement. Polyethylene water bottles were cleaned weekly.

The green tea is placed in the iron pipe from the open side. Before that, making sure to close the water tap connected to the other side and open the power point and make sure that the voltage difference is set to the required value by the voltage regulator as well as fix the required number of rolls and open the device and measure the magnetization period Timing I used the time in my experiment 60 seconds and then we open the faucet to collect the water in the drip and measure the magnetic flux by gauss meter. Magnetism happens by directing all the green tea molecules towards the field that is placed on the iron tube where the green tea is inside. The electromagnetic field is surrounded by all the green tea molecules where the electromagnetic field effect is stronger than magnetization by magnets where the magnetization is weaker because it only moves the molecules located on the boundary of the field as showed in Figure (4). 

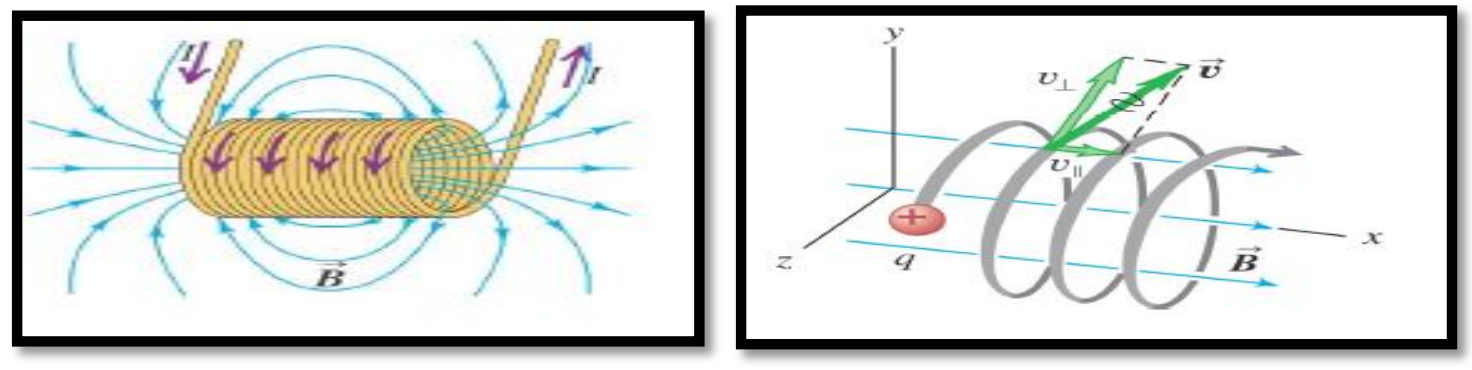

Figure (4): Electromagnetic field effect

Evaluation of Bone Densitometry By Using DXA (BMD and BMC)

DXA is the best method of measuring bone density and, thus, the best available indicator of fracture risk. Plain radiographs of supplement to DXA when there is a specific reason for the use. A number of methods for measuring BMD have been developed. Radiographic absorptiometry has not found widespread use, although the recent development of sophisticated computer analyses has renewed interest in this technique and may lead to its re-evaluation [22-7].

Measurement of "bone mineral density (BMD)" by central dual-energy. "X-ray absorptiometry (DXA)Central DXA" actually measures "bone mineral content (BMC)" and an area of bone, and it uses these numbers to calculate" BMD" [20]. Different types of "BMD" tests, all are non-invasive. Most tests vary according to which bones are measured, to establish the" BMD" and "BMC" results which varies by age, race and gender.

Z-score:- is serious for the interpretation of "BMD" measurements in clinical research and practice. That can be recognized in part to generalization of the relevance of" BMD" values to risk of fracture and involvement threshold, and parallel to risk gradients factors in other widespread clinical diseases such as" heart attacks and stroke". the diagnosis of "low bone mass for age" is the categorization of choice, if the Z-scores is $(\backslash$ 2.0)in these groups, in the suitable clinical context [20].

Determining how the individual evaluate to their peers. The density measured according to" Z-score", the number of standard deviations(SD) below or above the mean for rats under treatment of exposed magnetized nutrition (green tea) as well as controlled group.

T-scores :-The T-score is the relevant measure when screening for osteoporosis. The criteria of the World Health Organization are [21] Normal:- is a T-score of $(-1.0)$ or higher, "Osteopenia" is defined as between $(-1.0$ and -2.5$)$.

Also "T-score", the number of standard deviations( SD) below or above the mean for rats under treatment of exposed magnetized nutrition The "T score" is used to analyze osteoporosis in older adults rats take ( magnetized water) in Tap (4200), as well as controlled group.

Results were generally achieve by two measures, the" T-score and the Z-score" . 
Scores be a sign of the amount one's "bone mineral density" varies from the mean. Negative scores: indicate (lower bone density), and positive scores: indicate ( higher bone density), in addition to Pelvis BMD and Pelvis BMC score.

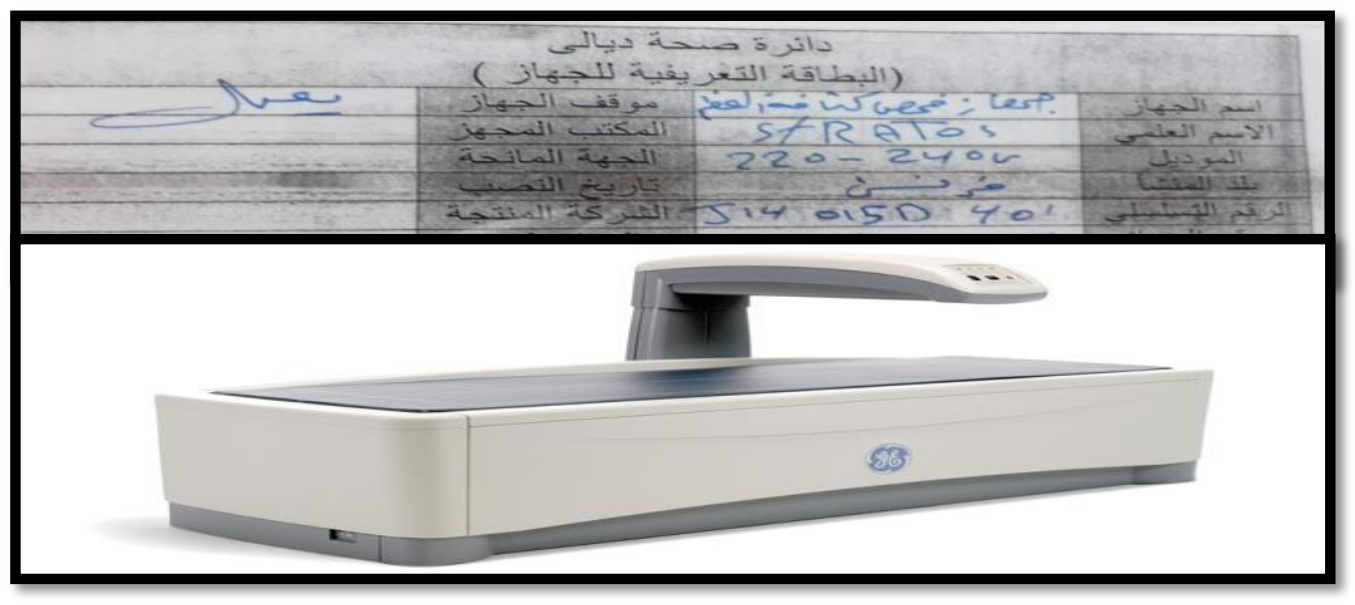

Figure (5): DXA X ray imaging technique measures Bone Densitometry.

\section{Protective measures}

Protective measures and personal hygiene to find if protective equipment,hygiene and knowledge of pollution risks have an impact on magnetic exposure in this study.

\section{Statistical analysis}

Results are presented as means with their standard error of the mean (mean \pm Str. Error of mean). Statistically analysis of data was carried out using the available statistical package of SPSS-02 (Statistical Package for Social Sciences version 2) .All data were tested for normality using the PROC UNIVARIATE, a procedure used mainly for examining the distribution of data. Homogeneity of group variances was estimated using Levine's test. The variety of group means were compared using one-way analysis of variance (ANOVA), followed by post-hoc Tukey's test for pair-wise multiple comparisons of the group means. The
Welch's variance-weighted ANOVA was used in place of the simple one-way ANOVA when the assumption of homogeneity of variances was not met and groups were unequal in size, which was followed by post hoc Tukey's test for pair-wise multiple comparisons. A difference was considered to be statistically significant when $p \leq 0.05$.

\section{Results}

Presented Results utilized from the experimental part of the study includes, impact of electromagnetic field experiment, and simultaneous action of intensity of bone turnover in rats, compares the efficiencies in each case as well as the spectral measurements. The gain achieved from the magnetization system has been analyzed in accordance with effectiveness of magnetized green tea.

The information collected in this section was further put to a statistical analysis. The 
results obtained are based on SPSS Statistics generates tables in one-way ANOVA analysis and Tukey post hoc test. The sample for the study was divided into four categories according to The ANOVA statistical evaluation of the mean values of group (control, water) and number of rolls (1400) as shown in Tables(2) bellow:

Left leg (BMD, BMC, T-score, Z-score ) Table(2) shows distribution of left leg (BMD, BMC, T-score, Z-score ) among study sample (Tea and control ) according to Mean, as appears below the Control Group (BMD = $0.04, \mathrm{BMC}=0.50, \mathrm{~T}$-score $=4.81, \mathrm{Z}$-score $=$ $5.00)$, the highest mean value in BMD green

Table (5): The distribution of left leg (BMD, BMC, T-score and Z-score) among study sample (Green tea and Control) according to Mean and stander error

\begin{tabular}{|l||l||l||l||l|}
\hline & left leg BMD & left leg BMC & $\begin{array}{l}\text { left leg T- } \\
\text { score }\end{array}$ & left leg Z-score \\
\hline \hline Green tea & $0.20 \pm 0.006$ & $28.54 \pm 3.959$ & $20.76 \pm 0.635$ & $20.95 \pm 0.603$ \\
\hline \hline Control & $0.04 \pm 0.001$ & $0.50 \pm 0.021$ & $4.81 \pm 0.208$ & $5.00 \pm 0.204$ \\
\hline \hline Total & $0.12 \pm 0.003$ & $14.52 \pm 1.99$ & $12.785 \pm 0.421$ & $12.975 \pm 0.403$ \\
\hline
\end{tabular}

* The mean difference is significant at the 0.05 level according Tukey test.

*Test treat between magnetized Green tea and control groups.

Distribution of left leg (BMD, BMC, Tscore, Z-score ) among magnetic fields 1400 according to Mean, as appears below the Control Group $(\mathrm{BMD}=0.04, \mathrm{BMC}=0.50, \mathrm{~T}$ score $=4.81$, Z-score $=5.00)$, the highest mean value in BMD exists 1400 roll (mean $=0.20$,Str. Error \pm 0.005$)$ the less value in the Control Group ( mean $=0.04$,Str. Error $\pm 0.001)$. According to BMC the highest mean value exists in 1400 roll (mean $=26.67$ ,Str. Error \pm 2.162 ) less value in the Control Group (mean=27.45, Str. Error \pm 4.058$)$. tea $($ mean $=0.20$, Str. Error \pm 0.006$)$. According to $\mathrm{BMC}$ the highest mean value exists in green tea (mean $=28.54$, Str. Error \pm 3.959$)$. While in $\mathrm{T}$-score the highest mean value exists in green tea $($ mean $=20.76$, Str. Error \pm 0.635$)$. While in Z-score the highest mean value exists in green tea (mean $=20.95$, Str. Error \pm 0.603$)$. According to the Analysis of ANOVA, in green tea group highest statistically significant difference appears in left leg - BMC, left leg - BMD, left leg - T-score and left leg - Zscore $\mathrm{p}<0.05$. 
Evaluation The Effect of Magnetized green tea extracts on The Bone Density And Osteoporosis In The Experimental Rats By DXA-Scan

Table (6): The distribution of pelvis (BMD, BMC, T-score and Z-score) among magnetic fields (1400 and control ) and control according to Mean and stander error

\begin{tabular}{||l||l|l||l|l||}
\hline & Left leg BMD & Left leg BMC & Left leg T-score & Left leg Z-score \\
\hline \hline 1400 roll & $0.20 \pm 0.005$ & $27.45 \pm 4.058$ & $20.47 \pm 0.732$ & $23.09 \pm 0.572$ \\
\hline \hline Control & $0.04 \pm 0.001$ & $0.50 \pm 0.021$ & $4.81 \pm 0.208$ & $5.00 \pm 0.204$ \\
\hline \hline Total & $0.12 \pm 0.003$ & $13.975 \pm 2.039$ & $12.655 \pm 0.47$ & $14.04 \pm 0.388$ \\
\hline
\end{tabular}

*The mean difference is significant at the 0.05 level according Tukey test.

*Test treat between 1400 rolls group and control group.

\section{Discussion}

Increasing evidence shows that magnetic fields and magnetic recipients that exposed to magnetic fields can play unique roles in promoting vital functions such as bone repair and regeneration. This study addresses the synergistic effects of magnetic filed treatment and groups of exposed nutrition's (green tea) due to response the bone regeneration in compared with Control group . Additionally, the exploration of using system of magnetization in order to create magnetic field makes the materials magnetized consist of (1400 rolls). Paramagnetic materials can response to external applied magnetic fields, showing small and positive susceptibility. The magnetic properties do not persist if the external magnetic field is removed. Regarding to the magnetic responsive property of super-paramagnetic nanoparticles, we particularly address ourselves to the synergy effect of magnetic scaffolds with magnetic fields on osteoblast cells [19].

In current study rats have treated daily for 21 days with magnetized green tea extract showed a significant increase in concentration of high density of bone (BMD, $\mathrm{BMC}, \mathrm{T}-$ score and $\mathrm{Z}$ - score) and given magnetized green tea every day. Tea drinking has been shown to be positively associated with bone mineral density [6]. DXA is the best method of measuring bone density and, thus, the best available indicator of fracture risk. Plain radiographs of supplement to DXA when there is a specific reason for the use. DXA should be repeated every 2 to 4 years for patients receiving ovarian hormone therapy and 1 to 2 years for patients undergoing bisphosphonate therapy. Measurements and reporting of results are standardized.

Reports refer to the World Health Organization's recommended definitions. Bone mineral density (BMD) is an important component of bone strength. Its measurement is considered the best single method for assessing future risk of osteoporotic fractures and for confirming the diagnosis of osteoporosis[24-1].

A number of methods for measuring BMD have been developed. Radiographic absorptiometry has not found widespread use, although the recent development of sophisticated computer analyses has renewed interest in this technique and may lead to its re-evaluation [23] [4]. 
Left leg (BMD,BMC,T-score and Z-score) due to nutrition and Magnetic Fields

Through out the above mentioned tables that show an increase in the concentrations of mineral components in the infusions was only linked with variable magnetic fields. It was observed high accuracy significant value of Left leg (BMD, BMC, T-score, Z-score ) among study sample (green tea) as well as Magnetic Fields (1400rolls) according to Mean , whereas Control Group (BMD = $0.04, \mathrm{BMC}=0.50, \mathrm{~T}$-score $=4.81, \mathrm{Z}$-score $=$ $5.00)$.

Thus highest mean value in BMD according to exists green tea (mean $=0.20$ ,Str. Error \pm 0.006 , while the highest mean value in BMD according to magnetic fields exists 1400 roll (mean $=0.20$,Str. Error \pm 0.005 ), BMC highest mean value according to magnetic fields exists in 1400 roll (mean= 27.45 ,Str. Error \pm 4.058 ), where T-score highest mean value due to green tea (mean= 18.76, Str. Error \pm 0.542 ), T-score highest mean value according to magnetic fields exists in 1400 roll $($ mean $=20.47$, Str.
Error \pm 0.732 ), while Z-score highest mean value due to green tea $($ mean $=20.95$, Str. Error \pm 0.702 ), Z-score the highest mean value according to magnetic fields exists in 1400 roll (mean= 23.09, Str. Error \pm 0.572 ). As for Post Hoc Test of groups according to green tea and 1400 rolls and control of left leg (BMD, BMC, T-score, Z-score) the distraction of post hoc tests showed that According to the Analysis of ANOVA show high accuracy statistically significant difference where $\mathrm{p}<0.05$.

\section{Magnetizing different kind of Tea ( green tea with mint extract)}

In current study, problematic action encountered when magnetizing another kind the green tea with mint extract ( different brand than what been used earliar of experiment ) rather than the original one , resulting changes at the Tap 1400 of tea color because of difference of longitudinal waves for some of the Tea compounds and after green tea analysis with FTIR Devise IR before and after magnetic process the following shaps appear:

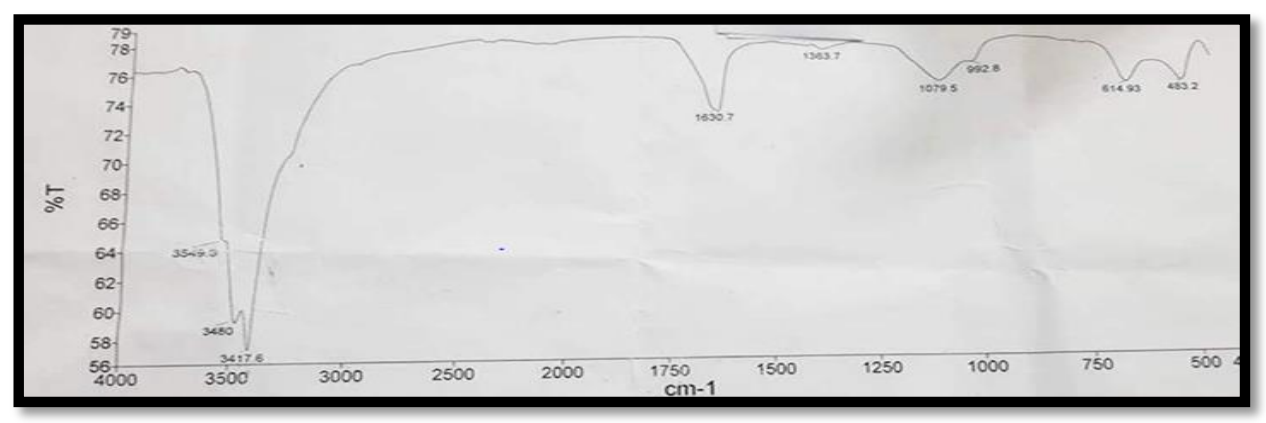

(a) 


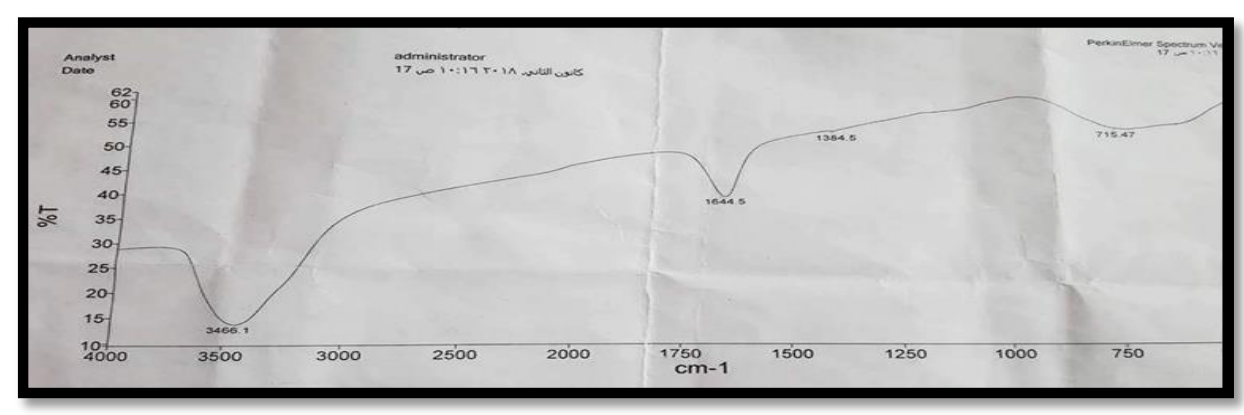

(b)

Figure (6): Shows before (a) and after(b) magnetic process shaps

Before magnetic process, on 3549.3 stretching has became after magnetic analysis with FTIR Devise IR 3466.1 stretching in which the bond $\mathrm{OH}$ Phenol stretched (stretching vibrations) for Tea structure contains five Hydroxide groups also galic acid component contains one phenol group. Spectrum radiology infrared reveals the Hydroxide groups that belong to Phenol components appearing in Spectrum between ( $3200-3600)$. At this point, the shift called hydpsochromic shift or in other words Blue Shift which is pack shift absorption to shorter wavelength as a result of compensating or solvent effect.

However, on 3480 \& 34176 stretching that $\mathrm{NH} 2$ bond stretch (stretching vibrations) exists in theanin disappears where the $\mathrm{H}$ in $\mathrm{NH} 2$ displaced and vanished. While before magnetic process on 1630.7 stretching has became after magnetic process on 1400 Tap 1644.5 stretching in which the bond $\mathrm{C}=\mathrm{N}$ stretched (stretching vibrations) that exists in caffeine component where the resulted displacement considered as a Red Shift ( Bathochromic Shift) which is pack shift absorption to longer wavelength as a result of compensating or solvent effect.

However, before magnetic process on 1363.7 stretching has became after magnetic process on 1384.5 stretching in which the bond $(\mathrm{C}-\mathrm{N})$ stretched (stretching vibrations) that exists in caffeine and theanin where the resulted displacement considered as a Red Shift ( Bathochromic Shift) which is pack shift absorption to longer wavelength as a result of compensating or solvent effect.

While on 1079.5 stretching resulte disappearance due to bond (C-O) stretched (stretching vibrations). As on 992.3 stretching after magnetic process became on 715.47 stretching in which the bond (C-O) stretched resulting hydpsochromic shift or the Blue Shift which is pack shift absorption to shorter wavelength as a result of or solvent effect.

\section{Conclusions}

In conclusion, the current study that represented our findings of the impact of electromagnetic field and also simultaneous action of those fields, on the intensity of bone turnover process in rats exhibited that 
magnetized green tea by using system of magnetization, stimulation partially preserves bone mass, .osteoporosis, and bone structure by promoting skeletal activities in experimental rats, which are revealed by reducing the bone-building duration to two weeks compared to the control group, which was in 24 days. the results of this study can be used for future clinical trial.

\section{References}

[1]Cummings SR, Black D. Bone mass measurements and risk of fracture inCaucasian women: a review of findings from prospective studies. Am i Med1995;98(2A):S24-8.

[2]Alhammer AH, Sadiq GT, and Yousif S. (2013). Effect of magnetized water on severalbiochemical and physicalproperties in mice. J Babylon Univ Pure ApplSci, 21(3):910-916.

[3]Attia YA, El-Hamid AE, El-Hanoun AM, Al-HarthiMA,Abdel-Rahman GM, and Abdella MM. (2015). Responses ofthe fertility, semen quality, blood constituents, immunity andantioxidant status of rabbit bucks to type and magnetizing ofwater. Annals Animal Sci, 15(2): 387-407.

[4]Genant HK, Engelke K, Fuerst T, et al. Noninvasive assessment of bonemineral and structure: state of the art. IBoneMinerRes 1996:11;707-30.

[5]Chaplin, M. (26 July 2011). "Descalingof Water" . Water Structure and Science. London South Bank University. Retrieved2012-03-26.

[6]Chang, C.J.; Chiu, K.L.; Chen, Y.L.; Chang, C.Y. Separation of catechins from green tea using carbon dioxide extraction. Food Chem. 2000, 68, 109-113. [CrossRef] [7]Genant HK, Engelke K, Fuerst T.,1996. Noninvasive assessment of bonemineral and structure: state of the art. IBoneMinerRes :11;707-30.

[8]Ikezoe, N.; Hirota, J.; Nakagawa, S. and Kitazawa, K. (1998).Making water levitate. Natural, 393: 749-750.

[9]"Interview of Klaus Kronenberg, Ph. D" .GMX International. Retrieved 2012-03-26.

[10]International Society for Clinical Densitometry. 2014 Facility Accreditation. Available at: http://www.iscd.org/ accreditation/. Accessed March 15, 2016. [11]Khudiar K, and Ali AM (2012). Effect of magnetic water on some physiological aspects of adult male rabbits. In Proceeding of the Eleventh Veterinary Scientific Conference Pp.120-126.

[12]Kelly, T.L., SLOVIK, D.M., NEER, R.M., (1989) . Calibration and standardization of bonemineral densitometers, J. Bone Miner. Res. 45 663669.

[13]Laskey, M., PHIL, D., (1996) Dualenergy $X$ ray absorptiometry and body composition,Nutrition12 1 -45-51.

[14]Macintyre, Steven A. 2014."Magnetic field measurement" (PDF). ENG Net Base References(2000). CRC Press LLC. Retrieved29 March 2014.

[15]National Research Council . Nutrient requirements of laboratory animals (4th revised ed.) Washington ,DC:National Academy Pree,pp3-79,1995. 
[16]National Research Council. Guide for the care and use of laboratory animal . Washington ,DC:National Academy Pree,pp1-79,1996.

[17]Neto GB, EngraciaFilho JR, de Oliveira BRSM, Coelho CMM,de Souza LFA, and Louzada MJQ. (2017). Water treatment bymagnetic field increases bone mineral density of rats. J ClinDensit. DOI: http://dx.doi.org/10.1016/j.jocd.2017.06.002. ). Kanis JA, Melton L III, Christiansen C, et al. The diagnosis of osteoporosis. Bone Miner Res 1994;9:1137-41.

[18]Parekh A. Decoding cytosolic Ca2+ oscillations. Trends Biochem. Sci. 2011;36:78-87.

[19]Meng J, Zhang Y, Qi X, et al. Paramagnetic nanofibrouscomposite films enhance the osteogenic responses of preosteoblastcells. Nanoscale, 2010, 2(12): 2565-2569Yacout MH, Hassan AA, Khalel MS, Shwerab AM,Abdel-Gawad EI, et al. (2015). Effect of Magnetic Water on thePerformance of Lactating Goats. J Dairy Vet Anim Res 2(5):00048. DOI: 10.15406/jdvar.2015.02.00048.

[20]The International Society for Clinical Densitometry,2016

[21]WHO Scientific Group on the Prevention and Management of Osteoporosis (2000 : Geneva, Switzerland) (2003). "Prevention and management of osteoporosis : report of a WHO scientific group" (pdf). Retrieved 2007-05-31 [22]Yates AJ, Ross PD, Lydick E, Epstein RS. Radiographic absorptiometry inthe diagnosis of osteoporosis [review]. Am J Med 1995;98(2A):S41-7.

[23]Yates AJ, Ross PD, Lydick E, Epstein RS. Radiographic absorptiometry inthe diagnosis of osteoporosis [review]. Am J Med 1995;98(2A):S41-7.

[24]Kanis JA, Melton L III, Christiansen C, et al. The diagnosis of osteoporosis.Bone Miner Res 1994;9:1137-41. 